\title{
Correction to: The association of stage 1 hypertension defined by the 2017 ACC/AHA hypertension guideline and subsequent cardiovascular events among adults $<50$ years
}

\author{
Sitong Liu ${ }^{1} \cdot$ Yali Wang ${ }^{1} \cdot$ Yanxia Xie ${ }^{1} \cdot$ Jia Zheng ${ }^{1} \cdot$ Rongrong Guo $^{1} \cdot$ Yue Dai ${ }^{1} \cdot$ Zhaoqing Sun $^{2} \cdot$ Liying Xing $^{3}$. \\ Xingang Zhang ${ }^{4} \cdot$ Yingxian Sun $^{2} \cdot$ Liqiang Zheng $^{1}$
}

Published online: 19 September 2019

(c) The Author(s), under exclusive licence to Springer Nature Limited 2019

Correction to: Journal of Human Hypertension https://doi.org/10.1038/s41371-019-0242-7
In the original Article, the multivariate analysis contained an error that affected its intended meaning. This has now been corrected in the PDF and HTML versions of this article.

The original article can be found online at https://doi.org/10.1038/ s41371-019-0242-7

Yingxian Sun

sunyingxian12@126.com

$\triangle$ Liqiang Zheng

liqiangzheng@126.com

1 Department of Clinical Epidemiology, Library, Shengjing Hospital of China Medical University, 110004 Shenyang, PR China

2 Department of Cardiology, Shengjing Hospital of China Medical University, 110004 Shenyang, PR China

3 Institute of Chronic Disease, Liaoning Provincial Center for Disease Control and Prevention, 110005 Shenyang, PR China

4 Department of Cardiology, The First Affiliated Hospital of China Medical University, 110001 Shenyang, PR China 